\title{
Sedation strategies in children with pediatric acute respiratory distress syndrome (PARDS)
}

\author{
Lynne Rosenberg, Chani Traube \\ Department of Pediatrics, Weill Cornell Medical College, New York, NY, USA \\ Contributions: (I) Conception and design: C Traube; (II) Administrative support: Department of Pediatrics, Weill Cornell Medical College; (III) \\ Provision of study materials or patients: None; (IV) Collection and assembly of data: All authors; (V) Data analysis and interpretation: All authors; (VI) \\ Manuscript writing: All authors; (VII) Final approval of manuscript: All authors. \\ Correspondence to: Chani Traube. Department of Pediatrics, Weill Cornell Medical College, New York, NY, USA. Email: chr9008@med.cornell.edu.
}

\begin{abstract}
In this review, we discuss the changing landscape of sedation in mechanically ventilated children with pediatric acute respiratory distress syndrome (PARDS). While previous approaches advocated for early and deep sedation with benzodiazepines, emerging literature has highlighted the benefits of light sedation and use of non-benzodiazepine sedating agents, such as dexmedetomidine. Recent studies have emphasized the importance of monitoring multiple factors including, but not limited to, sedation depth, analgesia efficacy, opiate withdrawal, and development of delirium. Through this approach, we hope to improve PARDS outcomes. Overall, more research is needed to further our understanding of the best sedation strategies in children with PARDS.
\end{abstract}

Keywords: Pediatric acute respiratory distress syndrome (PARDS); sedation; analgesia; delirium

Submitted Apr 16, 2019. Accepted for publication Aug 26, 2019.

doi: $10.21037 /$ atm.2019.09.16

View this article at: http://dx.doi.org/10.21037/atm.2019.09.16

\section{Introduction}

Acute respiratory distress syndrome (ARDS) was first described in adults in 1967 (1). The ARDS definition has since been modified to the current syndrome characterized by the Berlin Criteria (2). Historically, pediatric intensivists were limited to using these adult criteria and guidelines in the care of their patients. In 2015, pediatric acute respiratory distress syndrome (PARDS) was formally defined by members of the Pediatric Acute Lung Injury Consensus Conference (PALICC) as the presence of hypoxemia and radiographic changes in the context of a new lung infiltrate occurring within seven days of a known insult (3). This insult occurs with histological changes including infiltration of inflammatory markers, alveolar edema, and disruption of alveolar capillary barriers (4). As recognition of PARDS as a clinically entity grew, so did research (5). While much of the literature on sedation and care of the mechanically ventilated patient is still centered on adults, there is an emerging body of literature in the pediatric world.

In the past, the primary sedation strategy in mechanically ventilated adults with ARDS was deep sedation within the first 48 hours of intubation. This sedative-hypnotic approach was thought to be optimal to control both pain and psychological sequelae of mechanical ventilation $(6,7)$. However, in 2012, numerous reviews emerged compiling data suggesting benefits of an analgo-sedative approach $(8,9)$. In the Clinical Practice Guidelines for adult intensive care unit (ICU) in 2013, Barr et al. recommended light target levels of sedation with an analgesia-first approach to sedation, as a growing body of literature from adult ICUs demonstrated the negative outcomes associated with deep sedation (10-12). This review will focus on optimal sedation strategies for mechanically ventilated children. Given the close relationship between sedation and analgesia, paralysis, mobilization and delirium, we will briefly discuss these other important considerations. 


\section{Sedation}

\section{Depth of sedation}

In the past, clinicians favored early deep sedation in mechanically ventilated patients, as it was thought to improve their oxygenation status, decrease pain, and minimize negative perception of experiences $(6,13)$. However, as more literature emerged, a shift toward a "less is more" approach began. In 2013, adult guidelines suggested light sedation might be associated with improvement in outcomes without increasing negative sequelae of increased physiologic stress (12). The Pediatric Acute Lung Injury Consensus Conference group has since concurred, recommending "minimal yet effective targeted sedation" with continued "monitoring, titration, and weaning" $(14,15)$. These goals were reiterated in the adult guidelines produced in 2018 (16). Both groups describe an "analgo-sedation" approach, using analgesics first and adding a sedative only when necessary $(14,16)$.

Studies have shown a survival benefit associated with light sedation in ARDS. A 2014 multicenter prospective cohort study in 45 adult ICUs enrolled 322 mechanically ventilated and sedated patients. Results suggested increased hospital mortality with deep sedation (OR 2.36; $95 \%$ CI: 1.31 to 4.25 ), with more ventilator days (7 vs. 5 days, $\mathrm{P}=0.041)$ and more tracheostomies performed (38.9\% vs. $22 \%, \mathrm{P}=0.001)$ in the cohort with deep sedation (10). This is consistent with findings from a recent multicenter longitudinal cohort study of 42 ICUs around the world to assess 180-day survival in 703 mechanically ventilated adults. Results showed a stark dose-dependent relationship between sedation intensity and increased risk of death (HR 1.29, $95 \%$ CI: 1.15 to $1.46 ; \mathrm{P}<0.001$ ), increased prevalence of delirium (HR 1.25, 95\% CI: 1.10 to 1.43 ; $\mathrm{P}=0.001$ ), and decreased chance of early extubation (HR $0.80,95 \%$ CI: 0.73 to $0.87 ; \mathrm{P}<0.001)(17)$. A 2018 systematic review and meta-analysis reviewed the literature on the outcomes of early deep sedation in critically ill adults. Light sedation was associated with improved outcomes, including decreased number of ventilator days (mean difference $-2.1,95 \%$ CI: -3.6 to $-0.5 ; \mathrm{P}=0.008$ ) and decreased number of ICU days (mean difference $-3.0,95 \%$ CI: -5.4 to -0.6 ; $\mathrm{P}=0.02)$. Overall, they found a lower rate of mortality with light versus deep sedation (OR 0.34, 95\% CI: 0.21 to 0.54; $\mathrm{P}<0.001)$. The pooled results consistently indicated improved outcomes with lighter sedation (18).

\section{Timing of sedation}

In a 2012 multicenter prospective longitudinal cohort study in 25 adult ICUs, Shehabi et al. explored the effect of early sedation on patient outcomes. They defined "early" as sedation within four hours of initiating mechanical ventilation. Because most randomized controlled trials randomize beyond the four-hour time period, this window is not often captured. Their results showed that early deep sedation both delays extubation and increases overall mortality. In their study, each Richmond Agitation Sedation Scale (RASS) assessment indicating deep sedation acted as a predictor for delayed extubation (HR 0.90, 95\% CI: 0.87 to $0.94, \mathrm{P}<0.001$ ), hospital death (HR $1.11,95 \% \mathrm{CI}: 1.02$ to $1.20 ; \mathrm{P}=0.01$ ), and 6-month mortality (HR 1.08, 95\% CI: 1.01 to $1.16 ; \mathrm{P}=0.026)(11)$.

\section{Route of administration}

Administration of both sedatives and analgesics can be via continuous infusion or via intermittent dosing. Theoretical advantages to continuous infusion include titratability, decreased infection risk, and maintenance of steady-state levels. Unfortunately continuous infusions also increase the risk of over-sedation and tolerance $(19,20)$. Despite this, a 2014 survey of pediatric ICUs showed markedly higher rates of utilization of continuous infusions for sedatives in the mechanically ventilated pediatric population as compared to intermittent dosing (20). Further research is needed to determine the benefits of intermittent $v s$. continuous sedative infusions in PARDS.

\section{Daily sedation interruption (DSI)}

DSI is the practice of discontinuing sedation once daily to allow the patient to "emerge"; sedation is resumed thereafter. Theoretically, this can minimize over-sedation and improve patient outcomes (21). While DSI has been largely used in the adult ICU patient population, it is not without its drawbacks. Though it was originally suggested for minimization of negative sedative effects, a randomized controlled trial in 2012 suggests that, when compared to protocolized sedation, there was no difference in duration of mechanical ventilation or ICU stay with DSI versus non-DSI in adults (22). Further, research shows there may be more hemodynamic changes in children being awoken from sedation, and DSI therefore must be used with 
caution (however, on a reassuring note, there has been no increase in accidental extubations associated with DSI) (23). A landmark randomized controlled pediatric trial found that DSI did not affect the number of ventilator-free days, length of sedation, or total dose of sedation, but did increase overall mortality and agitation episodes in children with critical illness (24). In 2014, Kudchadkar et al. suggest that intermittent dosing of sedative medications may actually negate the need for sedation interruption at all while also decreasing exposure to sedative agents $(20,21)$. On balance, the current pediatric evidence strongly suggests that best practice may favor a light-handed approach to sedation throughout the child's course of mechanical ventilation (rather than deeper sedation with daily interruption) (24-26).

\section{Protocolized sedation}

Sedation protocols have been widely discussed in recent literature. In the adult population, Devlin et al. supported nursing-protocolized strategies to try and achieve light sedation levels, but this is still up for debate in children (16). In 2011, Deeter et al. successfully implemented a sedation protocol in a pediatric ICU (PICU) allowing for decreased use of benzodiazepines and opioids (26). However, in a 2014 survey, only $27 \%$ of pediatric intensivists utilized a treatment algorithm when working with sedated patients, and $52 \%$ of those protocols were physician-driven as opposed to nursing-led (20). The efficacy of protocolized sedation itself in children is uncertain. In 2015, Curley et al. conducted a randomized clinical trial to assess for efficacy of protocolized sedation (using a benzodiazepinebased sedation algorithm) in pediatric patients and found no difference in number of mechanical ventilation days, and found that patients on the sedation protocol had more days with higher pain scores and agitation. Of note, there were potentially beneficial secondary outcomes; patients on a sedation protocol in this study had fewer days of concomitant opioid use, overall fewer sedative class exposures, increased time awake during intubation, and fewer pressure ulcers (6). In contrast, two single-center PICU studies showed clinical benefits to nurse-driven protocolized sedation-it is noteworthy that both of these studies utilized a benzodiazepine-sparing sedative approach, and targeted light sedation levels $(25,26)$. Further research will be necessary to assess the effect of protocolized analgosedation in the pediatric population.

\section{Sedative choice}

In the past, benzodiazepines were the most common class of sedative used in mechanically ventilated children. In 2014, Kudchadkar et al. found significant heterogeneity in choice of sedative agents in pediatric ICUs, with a majority using midazolam for sedation, most commonly via continuous infusion. At that time, many hospitals restricted dexmedetomidine use, and less than $1 \%$ of respondents reported using dexmedetomidine alone for sedation (20). However, emerging pediatric literature suggests the benefits of $\alpha_{2}$-agonist use, including an association with decreased use of opioids and benzodiazepines, and improvement in pediatric ICU outcomes (27-31). Here we will discuss the possible benefits and drawbacks of dexmedetomidine versus benzodiazepines.

\section{Dexmedetomidine}

Recent literature suggests numerous possible benefits of dexmedetomidine and other $\alpha_{2}$-agonists. In a randomized clinical trial in 2009, Riker et al. found a significant decrease in negative outcomes such as duration of mechanical ventilation and delirium rates (32). In a landmark randomized controlled trial, Pandharipande et al. compared dexmedetomidine to lorazepam in critically ill adults, and multiple outcomes all favored the use of the $\alpha_{2}$-agonist. This study showed dexmedetomidine was significantly easier to control in achieving sedation goals, and was associated with lower delirium rates. There were also trends toward decreased mortality when comparing dexmedetomidine to lorazepam (33). The ease of sedation control was further validated in 2014 when Tanaka et al. showed that dexmedetomidine use alone was unlikely to result in over-sedation, when compared to use of midazolam and fentanyl (10). Additionally, the sleep profile of dexmedetomidine has been shown to be superior to other sedative analgesics, with preservation of stage II sleep in a pediatric cohort (34). Finally, multiple studies have also reported on the opiate-sparing effects of dexmedetomidine, with decreases in opioid consumption when being coadministered with an $\alpha_{2}$-agonist (27-31).

Possible adverse effects associated with dexmedetomidine have been noted in case reports, including adrenal insufficiency, and acute discontinuation syndrome. Tachycardia, hypertension, and withdrawal are commonly reported side effects, although generally not clinically significant (31,35-37). Another significant barrier to use 
of dexmedetomidine is the cost; however, it is important to note that improved PICU outcomes such as decreased length of stay and decreased incidence of delirium likely results in significant cost-savings with the use of dexmedetomidine as first-line sedative $(33,38)$.

\section{Lack of pediatric evidence}

As dexmedetomidine has only recently been adopted for use in mechanically ventilated children, there is limited clinical research that has been published to date. In 2007, Tobias et al. conducted a literature review and found that dexmedetomidine had been used in a large number of pediatric patients with positive sedative and analgesic effects and limited negative effects (39). A single-center retrospective study from 2014 included 98 children and showed no significant hemodynamic effects that would limit long-term use in the PICU (31). Secondary analysis of data from a sedation titration trial showed that time at target sedation level was increased with use of dexmedetomidine ( $28 \%$ to $50 \%$ ), and cumulative opiate exposure was decreased (40). Hayden et al. conducted a review in 2016 to compile all the literature on $\alpha_{2}$-agonist efficacy in pediatric sedation. The findings were equivocal, as the paucity of research in this area led to lack of necessary power to draw statistical conclusions (41).

The SLEEPS Study (Safety profile, Efficacy and Equivalence in Paediatric intensive care Sedation study) enrolled 129 children in ten PICUs across England, and compared intravenous clonidine to midazolam for sedation in children on invasive mechanical ventilation. Although underpowered, this study was able to demonstrate noninferiority of clonidine (42). As such, while results for alpha agonist use in adults and children look promising, there is a need for more research to determine effect on pediatric outcomes.

\section{Benzodiazepines}

Despite their wide use in adults and children in the past, benzodiazepines have been associated with a number of negative effects. In December 2016, the Federal Drug Administration (FDA) released a warning regarding use of specific sedative agents in children under three years old, including lorazepam and midazolam, with a new blackbox warning (43). This was based on numerous animal and human studies that demonstrated neuronal loss, and effects on long-term cognitive outcomes (44-49). However many experts believe that this FDA announcement was premature; a perspective in the New England fournal of Medicine detailed how evidence from the FDA warning was incomplete, and that many relevant clinical studies were under way at the time of the release (50).

In support of the FDA warning, there is mounting clinical evidence that favors avoidance of benzodiazepines in the PICU setting. Benzodiazepines have been shown to frequently reach a deeper sedation than the initial target (when compared to dexmedetomidine), and deep sedation has been linked to poor outcomes (33). A 2012 prospective cohort study noted that midazolam exposure was associated with delayed extubation (11). Benzodiazepine exposure has also been linked to lower likelihood of ICU discharge in children (51). A further issue with benzodiazepines is their effect on sleep, with decreased REM and slow wave sleep (20,52-54).

In the pediatric world specifically, recent studies have elucidated the relationship between benzodiazepine use and development of delirium. In a prospective longitudinal cohort study, benzodiazepine administration was an independent predictor of delirium development in 1,547 critically ill children (adjusted OR 5.2, 95\% CI: 3.7 to 7.5; $\mathrm{P}<0.001)$ (55). A cohort study assessing delirium incidence and risk factors in 319 admissions of pediatric oncology patients found that receipt of benzodiazepines was associated with a near-quadrupling of delirium risk (OR 3.71; $\mathrm{P}<0.001$ ) (56). An international point prevalence study assessing the risk factors for delirium in 994 pediatric patients also showed benzodiazepine administration was an independent risk factor for development of delirium (adjusted OR 2.2, 95\% CI: 1.5 to 3.3; $\mathrm{P}<0.001)(57)$. Mody et al. took the association one additional step when they identified a temporal relationship and a dose response between benzodiazepine administration and development of delirium, citing a $43 \%$ increase in risk for subsequent delirium for every one $\log$ increase in benzodiazepine administration (58). Alvarez et al. further established this relationship as significant in an observational study in the pediatric cardiac intensive care unit (CICU) (59).

\section{Monitoring of sedation}

When working with sedated patients, it is highly important to both determine a goal sedation level and be able to monitor the sedation level frequently (Figure 1). In adult clinical guidelines, the Richmond Agitation-Sedation Scale (RASS) and Sedation-Agitation Scale (SAS) are considered the most valid and reliable tools for monitoring sedation levels in critically ill patients (12). Unfortunately, 


\begin{tabular}{|ll|}
\hline Monitoring Tools \\
\hline Analgesia & $\begin{array}{l}\text { Premature Infant Pain Profile (PIPP) } \\
\text { COMFORT } \\
\text { Face, Legs, Activity, Cry, Consolability } \\
\text { (FLACC) } \\
\text { Multidimensional Assessment of Pain } \\
\text { Scale (MAPS) }\end{array}$ \\
\hline Delirium & $\begin{array}{l}\text { Richmond Agitation Sedation Scale } \\
\text { (RAS) } \\
\text { State Behavioral Scale (SBS) }\end{array}$ \\
\hline $\begin{array}{l}\text { Cornell Assessment of Pediatric } \\
\text { Delirium (CAP-D) } \\
\text { Pediatric Confusion Assessment } \\
\text { Method for the ICU (pCAM-ICU) }\end{array}$ \\
\hline
\end{tabular}

Figure 1 Our review of the literature urges the use of goal directed care. In order to optimize sedation in PARDS we need to monitor pain, sedation, and delirium prospectively and adjust our interventions accordingly. By setting measurable goals, using validated tools, and titrating to those goals, clinicians will be able to decrease their use of sedatives and analgesics and decrease the prevalence of delirium. PARDS, pediatric acute respiratory distress syndrome.

when surveyed in 2014 , only $42 \%$ of pediatric intensivists reported routinely using sedation scoring mechanisms in their PICUs (20). Since that time, the RASS was validated for use in a pediatric population, providing a quick and easy way to monitor sedation levels in all PICU patients (60). There has not been data since that time on the frequency of sedation scoring protocols, but given the shift in paradigm toward light sedation, this review would urge the use of monitoring to ensure the desired level is met. This is consistent with ESPNIC recommendations to use validated sedation assessment tools in critically ill children to avoid negative outcomes (61).

\section{Analgesia}

Analgesia is a highly important component of successful sedation and positive ICU outcomes (23). Recent studies suggest that pain is under-recognized and undertreated in ICU settings (62). Getting control of acute pain is highly important, as delay in pain relief can later transition to chronic pain and cause significant psychological sequelae such as anxiety and post-traumatic stress disorder (PTSD) $(63,64)$. It has been noted that over-sedation can lead to under-recognition of pain, with delay in treatment leading to suboptimal pain control (62). With an analgo-sedation approach, the patient will be more awake. With less sedative on board, the bedside providers will be more effective at recognizing and treating pain (Figure 2). Here we will discuss analgesic choice, route of administration, and monitoring strategies.

\section{Analgesic choice}

\section{Non-opioids}

Clinical guidelines in adults suggest multiple modalities to treat pain in mechanically ventilated critically ill adults, including but not limited to relaxation techniques, cold therapy, music therapy, massage, NSAIDs, neuropathic pain medication, ketamine, nefopam, and acetaminophen $(12,13,16)$. Though potentially beneficial, ketamine has had deleterious neurologic effects in animal studies, urging caution when used for long-term sedation or analgesia in children (65).

\section{Opioids}

In 2014, Kudchadkar et al. found significant heterogeneity in choice of analgesic agents in pediatric ICUs, with most using fentanyl for opioid analgesia and most commonly on continuous infusion (20). Tanaka et al. found a similar pattern of use in adults, with $39.4 \%$ of participants using midazolam and fentanyl together, and $12.4 \%$ using fentanyl alone (10). While opioids have a number of unwanted side effects including gastrointestinal upset and motility issues, they provide excellent pain control and can be used effectively in a PICU setting (13). If using an opioid, it seems that morphine may have a more favorable profile than fentanyl, as it requires less up-titration when dosing (66). Further research, with attention to pharmacokinetics and pharmacogenomics, will be necessary to determine optimal analgesic choice in children with PARDS. 


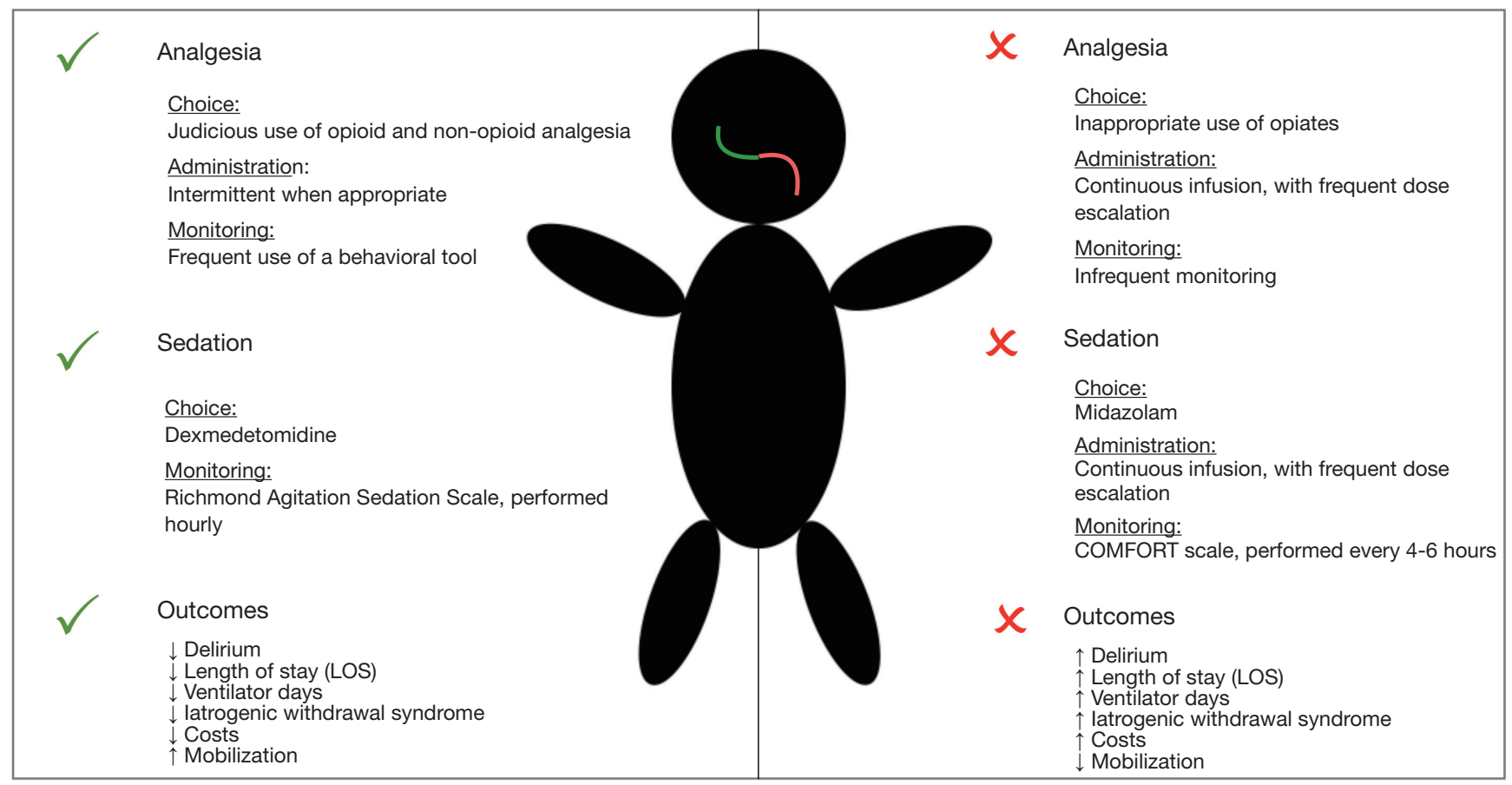

Figure 2 This cartoon provides an example of an analgo-sedation approach (on left) as compared to a traditional benzodiazepine-based sedation regimen.

\section{Route of administration}

Like sedatives, administration of analgesics can also be via continuous infusion or via intermittent dosing. Advantages of continuous infusion include titratability and maintenance of steady-state levels; however, there is the risk of increasing tolerance $(19,66)$. Of note, research suggests continuous infusions of opioids do not provide significant benefit over intermittent use in adults or children (67-69). Despite this evidence, Kudchadkar et al.'s review in 2014 suggested increased prevalence of continuous infusions for analgesics in the mechanically ventilated pediatric population (20). Further research may demonstrate benefit to an intermittent approach to opiate administration.

\section{Monitoring of pain}

Devlin et al. suggests that vital signs may not be a useful tool to monitor pain in mechanically ventilated patients. Instead, behavioral scales and self-reports are the most reliable measures $(12,16)$. There are a number of validated assessment tools for children that have been recommended by the European Society of Pediatric and Neonatal Intensive Care (EPNIC), including the Premature Infant Pain Profile (PIPP), the COMFORT scale, the Multidimensional Assessment of Pain Scale (MAPS), and the Face, Legs, Activity, Cry, Consolability scale (FLACC) (Figure 1) (61,70-73).

An important consideration when using analgesics in any child is the possibility of tolerance, withdrawal, and dependency. Literature suggests the development of tolerance is unlikely with less than 72 hours of therapy, and that iatrogenic withdrawal syndrome (IWS) does not generally develop unless opiates are used for six or more days $(61,74)$. After five days of exposure, it may be prudent to monitor for IWS when weaning medication. The Withdrawal Assessment Tool (WAT) can be used in mechanically ventilated children (75). In order to decrease the incidence of tolerance and withdrawal, Anand et al. recommend finding the minimum effective doses and avoiding long acting opioids if possible. The use of multiple drug classes in a rotation has been shown to be effective in adults, and may be considered for children who will be getting long-term opioid treatment $(19,23)$. It is worth noting that neonates and infants may develop tolerance to opioids more quickly, though the pathophysiology behind this phenomenon is not completely understood $(23,74)$. It is possible that these young children presented with delirium 
that was mis-diagnosed as "early tolerance" (as, without routine screening, delirium often goes undetected and mismanaged) (76).

\section{Paralysis/neuromuscular blockade (NMB)}

NMB can be a useful adjunct in a patient with severe PARDS. It can minimize ventilator-induced lung injury (VILI), prevent dyssynchrony, decrease trans-pulmonary pressures, and decrease oxygen consumption (77-83). However, NMB must be used sparingly, and with caution.

In 2009, a systematic review suggested NMB in ventilated adults might be a risk factor for later development of neuromuscular weakness. However, the authors noted that there was potential for many confounding factors in the studies reviewed, including co-administration of steroids (84). Papazian et al. conducted a seminal multicenter double blind trial in 2010 in mechanically ventilated adults with ARDS comparing use of cisatracurium besylate with placebo. This study showed that use of NMB for 48 hours resulted in decreased ventilator time, increased 90-day survival, and no increase in resulting neuromuscular weakness (80). This is consistent with numerous studies in the past decade that have shown no independent association between NMBs alone and muscular weakness or impaired muscle membrane excitability; rather, studies only show an association when co-administrated with steroids (80,85-89). However, a follow-up study executed by the NIH Prevention and Early Treatment of Acute Lung Injury (PETAL) network, "ROSE: Reevaluation Of Systemic Early Neuromuscular Blockade", was recently completed to assess the efficacy of NMBs in ARDS in adults. This large-scale randomized controlled trial $(n=1,006)$ showed no benefit to the use of early paralytic agents for moderate-to-severe ARDS with respect to barotrauma or mortality (while there were more adverse cardiovascular events in the paralyzed patients) (90). Experts have opined that this may be due to the deep sedation used in the first study (80), which may have increased the incidence of reverse triggering in the non-paralyzed cohort, and worsened VILI (91).

In 2016, Wilsterman et al. studied the short-term effects of NMBs in children and found improvement in the oxygenation index 15 minutes after administration. However, this study did not assess long-term outcomes such as VILI, barotrauma, or survival (92). There is inadequate research to conclude that NMBs are effective in pediatric respiratory failure. Further studies will be necessary to explore the potential benefit or harm of deeper sedation and/or paralysis in children with severe PARDS. As of now, PALICC recommends that only "if sedation alone is inadequate to achieve effective mechanical ventilation, neuromuscular blockade (NMB) should be considered" at the "minimal yet effective" dose $(14,15)$.

\section{Delirium}

In the fifth edition of the Diagnostic and Statistics Manual (DSM-V), the American Psychiatric Association defines delirium as a fluctuating disturbance of consciousness or change in cognition developing over a short period of time (93). As described above, poor sedation strategies can lead to delirium, which is independently associated with pediatric morbidity and mortality. This relationship makes delirium an important topic to address when discussing sedation approaches.

\section{Delirium effect on outcome}

Delirium can be highly problematic in children, leading to prolonged mechanical ventilation, increase in PICU and hospital length of stay, higher number of sequelae post-discharge, and increased morbidity and mortality $(12,51,55,57,59,94-97)$. Despite this, a 2014 review suggests the prevention, recognition, and treatment of delirium is inadequate in most pediatric intensive care units (20).

\section{Delirium risk factors}

There are numerous risk factors associated with delirium. Predisposing factors include young age and developmental disabilities $(51,55,59,95)$. While these risk factors are not modifiable, environmental hospital factors-such as poor sleep quality due to light and sound pollutionoften are. Sadly, as of 2014 , only a very limited number of pediatric ICUs optimized these factors, with a study showing that only $16 \%$ and $9 \%$ addressed noise and light exposure, respectively (20). However, it is likely that the single most important modifiable risk factor for delirium in mechanically ventilated children is sedative choice. As discussed in the sedation section above, sedation depth and exposure to benzodiazepines are highly associated with increased delirium risk $(12,51,55,57,58)$.

\section{Prevention of delirium}

Prevention begins with recognition of risk factors and 
implementation of monitoring tactics. To prevent delirium in adults, guidelines suggest "non-pharmacologic intervention focused on (but not limited to) reducing modifiable risk factors for delirium, improving cognition, and optimizing sleep, mobility, hearing, and vision in critically ill adults." $(12,16)$.

Regular assessment for delirium using a validated screening tool is highly important in the prevention, recognition, and treatment of delirium (16). In a 2014 survey, $71 \%$ of PICUs reported no routine delirium screening (20). That same year, the Cornell Assessment of Pediatric Delirium (CAPD) was developed and validated to assess for delirium in children of all ages. Importantly, the CAPD was specifically designed for ease of use in mechanically ventilated children (98). Another valid and reliable pediatric delirium screening tool is the Pediatric Confusion Assessment Method for the ICU (pCAM-ICU) for children older than 5 years old, with a preschool version available for children between 6 months and 5 years of age $(99,100)$. The EPNIC has recommended use of the CAPD for delirium screening in all critically ill children, and the CAPD is now used as standard of care in many North American PICUs $(61,101)$. In fact, studies have shown that simply implementing universal delirium screening can lead to a decrease in delirium rates (Figure 1) (25). Most relevant to PARDS, data show that less sedation will result in less delirium in mechanically ventilated children, and may lead to better short- and long-term outcomes (55).

\section{Mobilization}

Early mobilization (EM) is defined as "an interdisciplinary, goal-directed therapy used to facilitate movement" in patients with ARDS (102). Given that a commonly cited issue in the literature is the development of neuromuscular weakness following mechanical ventilation, intensivists began the routine use of mobilization to try and prevent its development (103).

Thus far, literature on the effects of mobilization has been overwhelmingly positive. Recent evidence suggests that EM in the ICU setting is not only safe, but also may improve functional outcomes (103). Clinical practice guidelines in adults suggest EM decreases both incidence and number of delirium days $(12,16)$.

In the pediatric world, an observational quality improvement project entitled "PICU Up!" studied 200 critically ill children to assess the feasibility of EM in the pediatric ICU. Post-implementation results demonstrated an increase in median number of mobilizations ( $3 \mathrm{vs}$. 6 ; $\mathrm{P}<0.001)$, without any mobilization related adverse events. This study advocated for the feasibility and safety of EM in children (104). As far as effectiveness of EM, a 2017 pediatric study implemented an EM protocol in the PICU and showed a significant decrease in delirium prevalence (25). As it is necessary for a patient to be awake and cooperative in order to participate in EM, this provides further incentive to adopt a light approach to sedation in PARDS.

\section{Conclusions}

Overall, mortality from PARDS has decreased over the last decade. With increased survival (specifically in higherincome countries), research has demonstrated substantial morbidity after PARDS. It is possible that a paradigm shift in our approach to sedation may further optimize the care we provide. With an analgo-sedation approach-targeting as light a level of sedation as possible (and using alternatives to benzodiazepines when sedation is required)—we may be able to decrease over-sedation, optimize pain control, shorten duration of mechanical ventilation, decrease delirium rates, and increase EM. A change in our approach to sedation may directly improve short- and long-term outcomes in PARDS.

\section{Acknowledgments}

None.

\section{Footnote}

Conflicts of Interest: The authors have no conflicts of interest to declare.

Ethical Statement: The authors are accountable for all aspects of the work in ensuring that questions related to the accuracy or integrity of any part of the work are appropriately investigated and resolved.

\section{References}

1. Ashbaugh DG, Bigelow DB, Petty TL, et al. Acute respiratory distress in adults. Lancet 1967;2:319-23.

2. ARDS Definition Task Force, Ranieri VM, Rubenfeld GD, et al. Acute respiratory distress syndrome: the Berlin Definition. JAMA 2012;307:2526-33.

3. Khemani RG, Smith LS, Zimmerman JJ, et al. Pediatric 
acute respiratory distress syndrome: definition, incidence, and epidemiology: proceedings from the Pediatric Acute Lung Injury Consensus Conference. Pediatr Crit Care Med 2015;16:S23-40.

4. Ware LB, Matthay MA. The acute respiratory distress syndrome. N Engl J Med 2000;342:1334-49.

5. Zimmerman JJ, Akhtar SR, Caldwell E, et al. Incidence and outcomes of pediatric acute lung injury. Pediatrics 2009;124:87-95.

6. Curley MAQ, Wypij D, Watson RS, et al. Protocolized sedation vs usual care in pediatric patients mechanically ventilated for acute respiratory failure: a randomized clinical trial. JAMA 2015;313:379-89.

7. Myhren H, Ekeberg O, Tøien K, et al. Posttraumatic stress, anxiety and depression symptoms in patients during the first year post intensive care unit discharge. Crit Care 2010;14:R14.

8. Devabhakthuni S, Armahizer MJ, Dasta JF, et al. Analgosedation: a paradigm shift in intensive care unit sedation practice. Ann Pharmacother 2012;46:530-40.

9. Bartel B. New sedation practices in the adult intensive care unit: analgosedation. S D Med 2012;65:234-5.

10. Tanaka LM, Azevedo LC, Park M, et al. Early sedation and clinical outcomes of mechanically ventilated patients: a prospective multicenter cohort study. Crit Care 2014;18:R156.

11. Shehabi Y, Bellomo R, Reade MC, et al. Early intensive care sedation predicts long-term mortality in ventilated critically ill patients. Am J Respir Crit Care Med 2012;186:724-31.

12. Barr J, Fraser GL, Puntillo K, et al. Clinical practice guidelines for the management of pain, agitation, and delirium in adult patients in the intensive care unit. Crit Care Med 2013;41:263-306.

13. Tobias JD. Acute pain management in infants and childrenPart 2: Intravenous opioids, intravenous nonsteroidal antiinflammatory drugs, and managing adverse effects. Pediatr Ann 2014;43:e169-75.

14. Pediatric Acute Lung Injury Consensus Conference Group. Pediatric acute respiratory distress syndrome: consensus recommendations from the Pediatric Acute Lung Injury Consensus Conference. Pediatr Crit Care Med 2015;16:428-39.

15. Valentine SL, Nadkarni VM, Curley MA, et al. Nonpulmonary treatments for pediatric acute respiratory distress syndrome: proceedings from the Pediatric Acute Lung Injury Consensus Conference. Pediatr Crit Care Med 2015;16:S73-85.
16. Devlin JW, Skrobik Y, Gélinas C, et al. Clinical practice guidelines for the prevention and management of pain, agitation/sedation, delirium, immobility, and sleep disruption in adult patients in the ICU. Crit Care Med 2018;46:e825-73.

17. Shehabi Y, Bellomo R, Kadiman S, et al. Sedation Intensity in the First 48 Hours of Mechanical Ventilation and 180Day Mortality: A Multinational Prospective Longitudinal Cohort Study. Crit Care Med 2018;46:850-9.

18. Stephens RJ, Dettmer MR, Roberts BW, et al. Practice Patterns and Outcomes Associated With Early Sedation Depth in Mechanically Ventilated Patients: A Systematic Review and Meta-Analysis. Crit Care Med 2018;46:471-9.

19. Tobias JD. Tolerance, withdrawal, and physical dependency after long-term sedation and analgesia of children in the pediatric intensive care unit. Crit Care Med 2000;28:2122-32.

20. Kudchadkar SR, Yaster M, Punjabi NM. Sedation, sleep promotion, and delirium screening practices in the care of mechanically ventilated children: a wake-up call for the pediatric critical care community. Crit Care Med 2014;42:1592-600.

21. Kress JP, Pohlman AS, O'Connor MF, et al. Daily interruption of sedative infusions in critically ill patients undergoing mechanical ventilation. N Engl J Med 2000;342:1471-7.

22. Mehta S, Burry L, Cook D, et al. Daily sedation interruption in mechanically ventilated critically ill patients cared for with a sedation protocol: a randomized controlled trial. JAMA 2012;308:1985-92.

23. Anand KJS, Willson DF, Berger J, et al. Tolerance and withdrawal from prolonged opioid use in critically ill children. Pediatrics 2010;125:e1208-25.

24. Vet NJ, de Wildt SN, Verlaat CW, et al. A randomized controlled trial of daily sedation interruption in critically ill children. Intensive Care Med 2016;42:233-44.

25. Simone S, Edwards S, Lardieri A, et al. Implementation of an ICU bundle: an interprofessional quality improvement project to enhance delirium management and monitor delirium prevalence in a single PICU. Pediatr Crit Care Med 2017;18:531-40.

26. Deeter KH, King MA, Ridling D, et al. Successful implementation of a pediatric sedation protocol for mechanically ventilated patients. Crit Care Med 2011;39:683-8.

27. Hünseler C, Balling G, Röhlig C, et al. Continuous infusion of clonidine in ventilated newborns and infants: a randomized controlled trial. Pediatr Crit Care Med 
2014;15:511-22.

28. Aydogan MS, Korkmaz MF, Ozgül U, et al. Pain, fentanyl consumption, and delirium in adolescents after scoliosis surgery: dexmedetomidine vs midazolam. Paediatr Anaesth 2013;23:446-52.

29. Prasad SR, Simha PP, Jagadeesh AM. Comparative study between dexmedetomidine and fentanyl for sedation during mechanical ventilation in post-operative paediatric cardiac surgical patients. Indian J Anaesth 2012;56:547-52.

30. Tobias JD, Berkenbosch JW. Sedation during mechanical ventilation in infants and children: dexmedetomidine versus midazolam. South Med J 2004;97:451-5.

31. Whalen LD, Di Gennaro JL, Irby GA, et al. Longterm dexmedetomidine use and safety profile among critically ill children and neonates. Pediatr Crit Care Med 2014;15:706-14.

32. Riker RR, Shehabi Y, Bokesch PM, et al. Dexmedetomidine vs midazolam for sedation of critically ill patients: a randomized trial. JAMA 2009;301:489-99.

33. Pandharipande PP, Pun BT, Herr DL, et al. Effect of sedation with dexmedetomidine vs lorazepam on acute brain dysfunction in mechanically ventilated patients: the MENDS randomized controlled trial. JAMA 2007;298:2644-53.

34. Mason KP, O’Mahony E, Zurakowski D, et al. Effects of dexmedetomidine sedation on the EEG in children. Paediatr Anaesth 2009;19:1175-83.

35. Tucker EW, Cooke DW, Kudchadkar SR, et al. Dexmedetomidine infusion associated with transient adrenal insufficiency in a pediatric patient: a case report. Case Rep Pediatr 2013;2013:207907.

36. Weber MD, Thammasitboon S, Rosen DA. Acute discontinuation syndrome from dexmedetomidine after protracted use in a pediatric patient. Paediatr Anaesth 2008;18:87-8.

37. Tobias JD. Dexmedetomidine: are tolerance and withdrawal going to be an issue with long-term infusions? Pediatr Crit Care Med 2010;11:158-60.

38. Traube C, Mauer EA, Gerber LM, et al. Cost associated with pediatric delirium in the ICU. Crit Care Med 2016;44:e1175-9.

39. Tobias JD. Dexmedetomidine: applications in pediatric critical care and pediatric anesthesiology. Pediatr Crit Care Med 2007;8:115-31.

40. Grant MJ, Schneider JB, Asaro LA, et al. Dexmedetomidine use in critically ill children with acute respiratory failure. Pediatr Crit Care Med 2016;17:1131-41.

41. Hayden JC, Breatnach C, Doherty DR, et al. Efficacy of $\alpha 2$-Agonists for Sedation in Pediatric Critical Care: A Systematic Review. Pediatr Crit Care Med 2016;17:e66-75.

42. Wolf A, McKay A, Spowart C, et al. Prospective multicentre randomised, double-blind, equivalence study comparing clonidine and midazolam as intravenous sedative agents in critically ill children: the SLEEPS (Safety profiLe, Efficacy and Equivalence in Paediatric intensive care Sedation) study. Health Technol Assess 2014;18:1-212.

43. Drug Safety and Availability $>$ FDA Drug Safety Communication: FDA review results in new warnings about using general anesthetics and sedation drugs in young children and pregnant women [Internet]. [cited 2019 Apr 3]. Available online: https://www.fda.gov/drugs/ drugsafety/ucm532356.htm

44. Jevtovic-Todorovic V, Hartman RE, Izumi Y, et al. Early exposure to common anesthetic agents causes widespread neurodegeneration in the developing rat brain and persistent learning deficits. J Neurosci 2003;23:876-82.

45. Wagner M, Ryu YK, Smith SC, et al. Review: effects of anesthetics on brain circuit formation. J Neurosurg Anesthesiol 2014;26:358-62.

46. Bong CL, Allen JC, Kim JT. The effects of exposure to general anesthesia in infancy on academic performance at age 12. Anesth Analg 2013;117:1419-28.

47. Block RI, Magnotta VA, Bayman EO, et al. Are Anesthesia and Surgery during Infancy Associated with Decreased White Matter Integrity and Volume during Childhood? Anesthesiology 2017;127:788-99.

48. DiMaggio C, Sun LS, Li G. Early childhood exposure to anesthesia and risk of developmental and behavioral disorders in a sibling birth cohort. Anesth Analg 2011;113:1143-51.

49. Flick RP, Katusic SK, Colligan RC, et al. Cognitive and behavioral outcomes after early exposure to anesthesia and surgery. Pediatrics 2011;128:e1053-61.

50. Andropoulos DB, Greene MF. Anesthesia and Developing Brains - Implications of the FDA Warning. N Engl J Med 2017;376:905-7.

51. Smith HAB, Gangopadhyay M, Goben CM, et al. Delirium and benzodiazepines associated with prolonged ICU stay in critically ill infants and young children. Crit Care Med 2017;45:1427-35.

52. Niedermeyer E, Lopes da Silva FH, Ovid Technologies, Inc. Electroencephalography: basic principles, clinical applications, and related fields. 5th ed. Philadelphia; London: Lippincott Williams \& Wilkins, 2005.

53. Kudchadkar S, Sterni L, Yaster M, et al. Sleep in the 
Intensive Care Unit. Contemp Crit Care 2009;7:1-12 .

54. Kudchadkar SR, Aljohani OA, Punjabi NM. Sleep of critically ill children in the pediatric intensive care unit: a systematic review. Sleep Med Rev 2014;18:103-10.

55. Traube C, Silver G, Gerber LM, et al. Delirium and mortality in critically ill children: epidemiology and outcomes of pediatric delirium. Crit Care Med 2017;45:891-8.

56. Traube C, Ariagno S, Thau F, et al. Delirium in Hospitalized Children with Cancer: Incidence and Associated Risk Factors. J Pediatr 2017;191:212-7.

57. Traube C, Silver G, Reeder RW, et al. Pediatric delirium in critically ill children: an international point prevalence study. Crit Care Med 2017;45:584-90.

58. Mody K, Kaur S, Mauer EA, et al. Benzodiazepines and development of delirium in critically ill children: estimating the causal effect. Crit Care Med 2018;46:1486-91.

59. Alvarez RV, Palmer C, Czaja AS, et al. Delirium is a common and early finding in patients in the pediatric cardiac intensive care unit. J Pediatr 2018;195:206-12.

60. Kerson AG, DeMaria R, Mauer E, et al. Validity of the Richmond Agitation-Sedation Scale (RASS) in critically ill children. J Intensive Care 2016;4:65.

61. Harris J, Ramelet AS, van Dijk M, et al. Clinical recommendations for pain, sedation, withdrawal and delirium assessment in critically ill infants and children: an ESPNIC position statement for healthcare professionals. Intensive Care Med 2016;42:972-86.

62. Sigakis MJ, Bittner EA. Ten myths and misconceptions regarding pain management in the ICU. Crit Care Med 2015;43:2468-78.

63. Kyranou M, Puntillo K. The transition from acute to chronic pain: might intensive care unit patients be at risk? Ann Intensive Care 2012;2:36.

64. Granja C, Lopes A, Moreira S, et al. Patients' recollections of experiences in the intensive care unit may affect their quality of life. Crit Care 2005;9:R96-109.

65. Li X, Li Y, Zhao J, et al. Administration of ketamine causes autophagy and apoptosis in the rat fetal hippocampus and in PC12 cells. Front Cell Neurosci 2018;12:21.

66. Anand KJS, Clark AE, Willson DF, et al. Opioid analgesia in mechanically ventilated children: results from the multicenter Measuring Opioid Tolerance Induced by Fentanyl study. Pediatr Crit Care Med 2013;14:27-36.

67. Bouwmeester NJ, Anand KJ, van Dijk M, et al. Hormonal and metabolic stress responses after major surgery in children aged 0-3 years: a double-blind, randomized trial comparing the effects of continuous versus intermittent morphine. Br J Anaesth 2001;87:390-9.

68. Salgado DR, Favory R, Goulart M, et al. Toward less sedation in the intensive care unit: a prospective observational study. J Crit Care 2011;26:113-21.

69. Penk JS, Lefaiver CA, Brady CM, et al. Intermittent versus continuous and intermittent medications for pain and sedation after pediatric cardiothoracic surgery; A randomized controlled trial. Crit Care Med 2018;46:123-9.

70. Merkel SI, Voepel-Lewis T, Shayevitz JR, et al. The FLACC: a behavioral scale for scoring postoperative pain in young children. Pediatr Nurs 1997;23:293-7.

71. Ramelet AS, Rees NW, McDonald S, et al. Clinical validation of the Multidimensional Assessment of Pain Scale. Paediatr Anaesth 2007;17:1156-65.

72. Wicksell RK, Renöfält J, Olsson GL, et al. Avoidance and cognitive fusion - central components in pain related disability? Development and preliminary validation of the Psychological Inflexibility in Pain Scale (PIPS). Eur J Pain 2008;12:491-500.

73. van Dijk M, Peters JWB, van Deventer P, et al. The COMFORT Behavior Scale: a tool for assessing pain and sedation in infants. Am J Nurs 2005;105:33-6.

74. Thornton SR, Smith FL. Characterization of neonatal rat fentanyl tolerance and dependence. J Pharmacol Exp Ther 1997;281:514-21.

75. Franck LS, Scoppettuolo LA, Wypij D, et al. Validity and generalizability of the Withdrawal Assessment Tool-1 (WAT-1) for monitoring iatrogenic withdrawal syndrome in pediatric patients. Pain 2012;153:142-8.

76. Ely EW, Siegel MD, Inouye SK. Delirium in the intensive care unit: an under-recognized syndrome of organ dysfunction. Semin Respir Crit Care Med 2001;22:115-26.

77. Tremblay LN, Slutsky AS. Ventilator-induced lung injury: from the bench to the bedside. Intensive Care Med 2006;32:24-33.

78. Marini JJ. Early phase of lung-protective ventilation: a place for paralytics? Crit Care Med 2006;34:2851-3.

79. Slutsky AS. Neuromuscular blocking agents in ARDS. N Engl J Med 2010;363:1176-80.

80. Papazian L, Forel JM, Gacouin A, et al. Neuromuscular blockers in early acute respiratory distress syndrome. $\mathrm{N}$ Engl J Med 2010;363:1107-16.

81. Forel J-M, Roch A, Marin V, et al. Neuromuscular blocking agents decrease inflammatory response in patients presenting with acute respiratory distress syndrome. Crit Care Med 2006;34:2749-57. 
82. Baron RM, Levy BD. Recent advances in understanding and treating ARDS. F1000Res 2016. doi: 10.12688/ f1000research.7646.1.

83. Heidemann SM, Nair A, Bulut Y, et al. Pathophysiology and management of acute respiratory distress syndrome in children. Pediatr Clin North Am 2017;64:1017-37.

84. de Jonghe B, Lacherade JC, Sharshar T, et al. Intensive care unit-acquired weakness: risk factors and prevention. Crit Care Med 2009;37:S309-15.

85. Weber-Carstens S, Deja M, Koch S, et al. Risk factors in critical illness myopathy during the early course of critical illness: a prospective observational study. Crit Care 2010;14:R119.

86. Sharshar T, Bastuji-Garin S, Stevens RD, et al. Presence and severity of intensive care unit-acquired paresis at time of awakening are associated with increased intensive care unit and hospital mortality. Crit Care Med 2009;37:3047-53.

87. Alhazzani W, Alshahrani M, Jaeschke R, et al. Neuromuscular blocking agents in acute respiratory distress syndrome: a systematic review and meta-analysis of randomized controlled trials. Crit Care 2013;17:R43.

88. Needham CJ, Brindley PG. Best evidence in critical care medicine: The role of neuromuscular blocking drugs in early severe acute respiratory distress syndrome. Can J Anaesth 2012;59:105-8.

89. Yehya N, Servaes S, Thomas NJ, et al. Corticosteroid exposure in pediatric acute respiratory distress syndrome. Intensive Care Med 2015;41:1658-66.

90. National Heart, Lung, and Blood Institute PETAL Clinical Trials Network, Moss M, Huang DT, et al. Early Neuromuscular Blockade in the Acute Respiratory Distress Syndrome. N Engl J Med 2019;380:1997-2008.

91. Slutsky AS, Villar J. Early Paralytic Agents for ARDS? Yes, No, and Sometimes. N Engl J Med 2019;380:2061-3.

92. Wilsterman MEF, de Jager P, Blokpoel R, et al. Short-term effects of neuromuscular blockade on global and regional lung mechanics, oxygenation and ventilation in pediatric acute hypoxemic respiratory failure. Ann Intensive Care 2016;6:103.

93. American Psychiatric Association. Diagnostic and

Cite this article as: Rosenberg L, Traube C. Sedation strategies in children with pediatric acute respiratory distress syndrome (PARDS). Ann Transl Med 2019;7(19):509. doi: 10.21037/atm.2019.09.16 statistical manual of mental disorders: DSM-V. 5th ed. American Psychiatric Association. Arlington, VA, 2013.

94. Smith HAB, Fuchs DC, Pandharipande PP, et al. Delirium: an emerging frontier in the management of critically ill children. Anesthesiol Clin 2011;29:729-50.

95. Silver G, Traube C, Gerber LM, et al. Pediatric delirium and associated risk factors: a single-center prospective observational study. Pediatr Crit Care Med 2015;16:303-9.

96. Pisani MA, Kong SY, Kasl SV, et al. Days of delirium are associated with 1-year mortality in an older intensive care unit population. Am J Respir Crit Care Med 2009;180:1092-7.

97. van den Boogaard M, Schoonhoven L, Evers AWM, et al. Delirium in critically ill patients: impact on long-term health-related quality of life and cognitive functioning. Crit Care Med 2012;40:112-8.

98. Traube C, Silver G, Kearney J, et al. Cornell Assessment of Pediatric Delirium: a valid, rapid, observational tool for screening delirium in the PICU. Crit Care Med 2014;42:656-63.

99. Smith HAB, Boyd J, Fuchs DC, et al. Diagnosing delirium in critically ill children: Validity and reliability of the Pediatric Confusion Assessment Method for the Intensive Care Unit. Crit Care Med 2011;39:150-7.

100.Smith HAB, Gangopadhyay M, Goben CM, et al. The preschool confusion assessment method for the ICU: valid and reliable delirium monitoring for critically ill infants and children. Crit Care Med 2016;44:592-600.

101. Traube C, Greenwald BM. "The Times They Are A-Changin": Universal Delirium Screening in Pediatric Critical Care. Pediatr Crit Care Med 2017;18:594-5.

102. Amidei C. Mobilisation in critical care: a concept analysis. Intensive Crit Care Nurs 2012;28:73-81.

103. Kress JP. Clinical trials of early mobilization of critically ill patients. Crit Care Med 2009;37:S442-7.

104. Wieczorek B, Ascenzi J, Kim Y, et al. PICU up!: impact of a quality improvement intervention to promote early mobilization in critically ill children. Pediatr Crit Care Med 2016;17:e559-66. 